\title{
Data support system for controlling decentralised nuclear power industry facilities through uninterruptible condition monitoring
}

\author{
Vladimir Povarov ${ }^{1}$, Aleksandr Danilov ${ }^{2}$, Viktor Burkovsky ${ }^{2}$ and Konstantin Gusev ${ }^{2 *}$ \\ ${ }^{1}$ Novovoronezh Nuclear Power Plant, 396072, Novovoronezh, Russia, \\ ${ }^{2}$ Voronezh State Technical University, 394026, Voronezh, Russia
}

\begin{abstract}
The article describes the automated uninterruptible multi-parameter system for monitoring operational vulnerability of critical NPP components, which differs from existing ones by being universally applicable for analysing mechanical damage of nuclear power unit components. The system allows for performing routine assessment of metal structures. The assessment of strained condition of a deteriorating component is based on three-dimensional finite element simulation with calculations adjusted with reference to in-situ measurements. A program for calculation and experimental analysis of maximum load and durability of critical area forms the core of uninterruptible monitoring system. The knowledge base on performance of the monitored components in different operating conditions and the corresponding comprehensive analysis of strained condition and deterioration rates compose the basis of control system data support, both for operating nuclear power units and robotic maintenance and repair systems.
\end{abstract}

\section{Introduction}

Rapid development of Information Technology extends its applicability and promotes intellectualisation of process management in every field of human activity. One of the most serious challenges is to provide information support by processing and analysing the information in the decision making process in managing decentralised nuclear power industry facilities. Nuclear Power Plant is a notable example of such facilities. Every power unit of a NPP is a dynamic facility and is under a high risk of emergency. Difficulties in controlling, monitoring and managing these power generation facilities are linked to the distance barriers between system elements and impossibility to describe their operation using standard modelling techniques. When managing nuclear facilities one will always face the issue of aggregating the results of assessing the current condition of NPP as a whole, as well as each of its subsystems (critical safety functions, parameters, readiness, etc.); aggregating or detailing the current information on any parameter group of elements and/or systems; detailing the information on any separate parameter, including current values, history and trends; diagnostic evaluation of power unit or equipment condition (operating mode); predicting power unit or equipment condition (operating mode); advising operating personnel where necessary. The uninterruptible on-line monitoring is essential due to the extensive use of automated repair systems in hardly accessible and highly radioactive elements of nuclear power units.

The main setback for this task was that the standard NPP design lacked systems for non-destructive damage control and actual thermal power load assessment of the most problematic equipment. During the operation of the nuclear power plants equipped with the VVER-type pressurised water reactors, uncompensated damage was identified, i.e. up to full depth cracks at welding joints of primary coolant header to steam generator connecting leg. Starting from 1980, inspections revealed dozens of in-service defects and even through-thickness cracks in pressuriser pipes, appeared due to stratification. It was discovered that a number of steam generators suffered similar damage; therefore the defects were recognised as a fundamental issue with a common cause.

Solving the problem required developing and introducing a new approach to calculation and experimental assessment of vulnerability of the most heavy-loaded elements of critical NPP equipment.

The present article describes the software and hardware complex for uninterruptible multi-parameter monitoring of condition of critical elements, susceptible to service damage by thermal distortion, as an integral part of the nuclear power unit operation control data support system.

\section{Problem formulation}

The analysis of accumulated experience in NPP operation revealed existence of critical elements (nodes, zones, junctions), susceptible to damage under heavy loads, including those beyond the design basis, as well as harmful environmental effects. It results in high risk of equipment integrity damage during the power operation due to a serious defect, such as coolant leak, with the possibility of further defect growth to the critical

*Corresponding author: gussev_konstantin@mail.ru 
dimension, i.e. resulting in the immediate threat to the power unit operation. In case of such safety-related and dynamically developing service defects, the top-priority task is finding root causes of defect formation and making management decisions in order to restore damaged element and prevent the recurrence of similar events in the future. To adopt a correct decision, the management needs to conduct the analysis of both retrospective and current data with visualisation of real element condition.

\section{Monitoring system}

To resolve the aforementioned problem, we propose the technical solution, based on the uninterruptible multiparameter monitoring and analysis of operational vulnerability of critical pieces of equipment, as the most effective way to visualise and control the formation and spread of defects in critical area. The main advantage of this system is its versatility, which allows for its' easy adaptation for monitoring almost any vulnerable zone of any component, including pipelines [1-4].

The practical result of the monitoring system implementation is the possibility for routine assessment of metal structures. Moreover, the use of the finite element simulation increases the accuracy of strain assessment, as calculations based on three-dimensional finite element simulation based on in-situ measurements are more precise, than approximationbased calculations.

All sensors are connected with the data collection and pre-processing unit, which in turn is connected to the data storage and transmission unit. The monitoring system is bundled with the software package that allows for automated remote system control, including data collection, storage and transmission, as well as real-time visualisation and express analysis of monitored parameters. The calculating module manages the threedimensional finite element simulation and allows for comparing data on formation and spread of operational defects with the current condition of the equipment and its' operational modes. You can find the structural diagram of the proposed system at Figure 1.

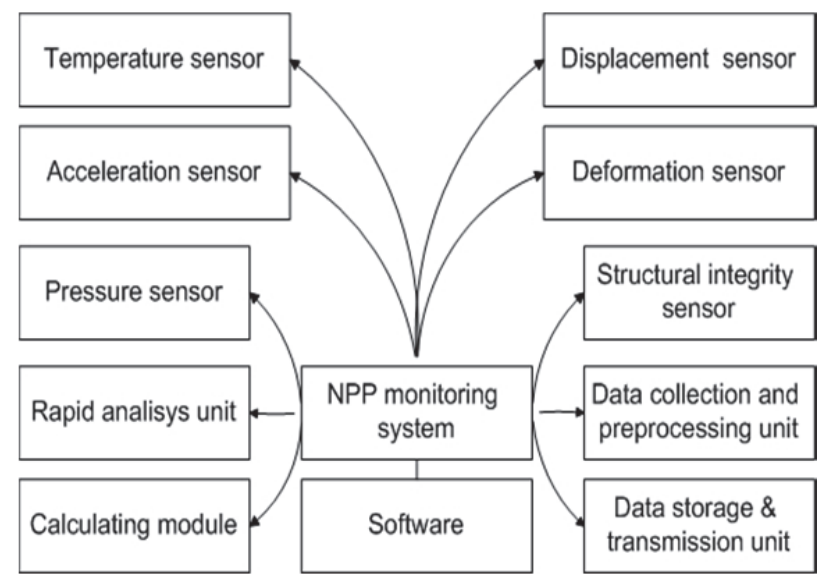

Fig. 1. Structural diagram of the uninterruptible multiparameter monitoring system.
Due to the multitude of tasks carried out by the uninterruptible multi-parameter monitoring complex and in order to unify separate units, the system can be built on multi-channel microprocessor measurement modules, including National Instruments-based, and assembled in one complex. The data storage and transmission unit is a high-performance industrial server for data storage and backup. The monitoring system remote control, including monitoring the environment, regular performance tests of the subsystems and measurement channels, as well as data collection, storage and transmission, is performed via software means through the data storage and transmission unit. All received data is automatically recorded and transferred to the remote analytical centre via the Internet for further processing and detailed analysis (Fig. 2).

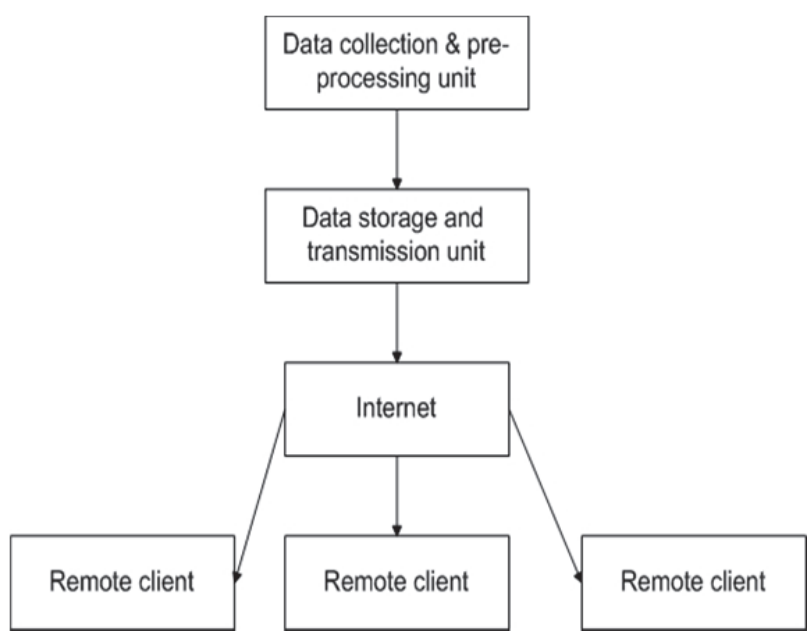

Fig. 2. Uninterruptible multi-parameter monitoring data transfer chart.

\section{Algorithm for calibrating the simulation}

Alongside the diagnostic data collecting, the uninterruptible monitoring system performs computational-experimental analysis of maximum load and durability of critical area based on the threedimensional finite element simulation. The software performing the procedure is the core of the system. The simulation has nodes duplicating actual sensor placement, which allows for the comparison of calculated and measured parameters. The key feature of the three-dimensional finite element simulation is possibility to use data collected through monitoring (temperature, deformation, displacement) as the actual source data, as well as test data for adjusting and calibrating the three-dimensional finite element simulation. The algorithm for calibrating the simulation is described in Figure 3 [3, 5-11]. The calibrating process is performed by comparing calculated and measured parameters (deformation, displacement, temperature in different operational modes and load levels).

The actual data on pressure and temperature, registered by regular reactor unit sensors, as well as 
uninterruptible monitoring system sensors, are used as the load parameters. The failure to achieve satisfactory correspondence of calculated and measured parameters is followed by adjusting the calculating programme, aligning the geometry of monitored object, condensing the finite element array in certain areas, correcting the boundary conditions, etc. Usually, correspondence of calculated and measured parameters is achieved after few such iterations, which indicates operational integrity of the simulation model and adequate simulation of loading during various operational modes.

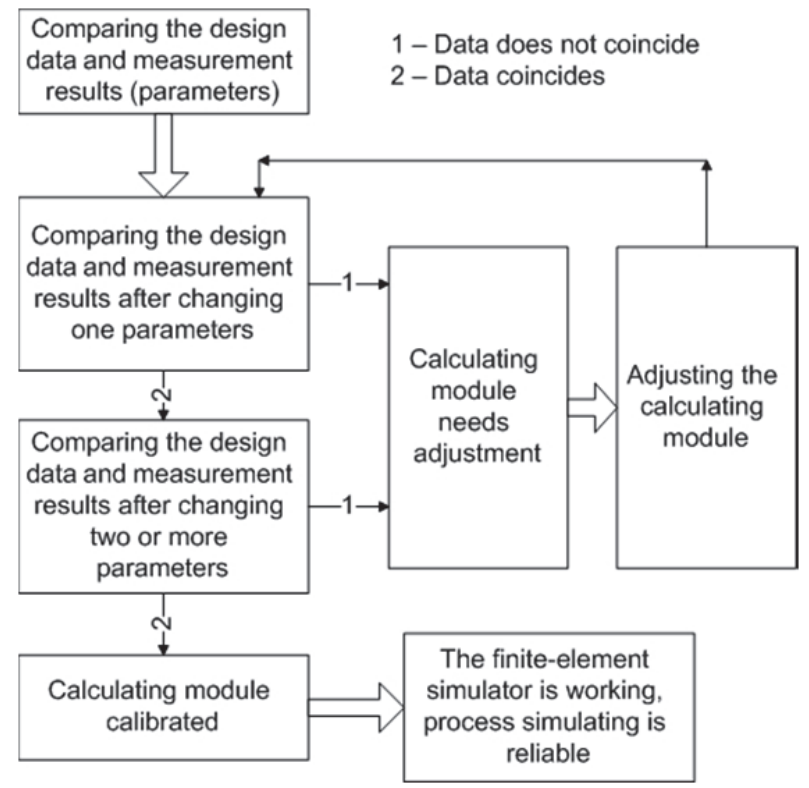

Fig. 3. The algorithm for calibrating the three-dimensional finite element simulation.

After the finite element simulation adjustment and calibration the calculation kernel is put into operation alongside the monitoring data collection process. The monitoring system enables real-time assessment of operational defect formation and advance kinetics, while calculation analysis allows for receiving stress distribution prospect at any time, based on actual load parameters. As a result, it helps to assess he actual accumulated damage rate with the fixed number of operational load cycles, as well as provide justified forecast for durability and service life of the monitored object.

With that said, all load history is collected during monitoring. Also, all events, noted by the monitoring system are compared to current operational modes, their characteristics and technological features. This allows identifying the link between the defect rate and certain factors and parameters.

\section{Monitoring algorithm}

On Figure 4 you can find a general algorithm for the multiparameter calculation and experimental monitoring. The main stages of the algorithm work are as follows. First, a three-dimensional finite element simulation model is developed and calculations for displacements and deformations in key nodes for main operational modes and various loading values are conducted. It is followed by the comparative analysis of calculated and experimental data with fixed coincidence error. In case of the successful result, the system is considered ready for monitoring, otherwise the necessary corrections are implemented for the finite element model (see Fig. 3). When the system is ready for operation, calculations for static strength and cyclic durability are performed consistent with the detected deficiencies and thermal power impact beyond the design basis. After that the causes of the latter are identified and compensatory actions are developed based on the set of condition-action rules.

\section{Conclusion}

The described system for uninterruptible monitoring is a multipurpose software and hardware complex for assessing the vulnerability of critical NPP equipment and predicting damage development at any physical impact parameters (i.e. pressure, temperature, vibration, movement, etc.) and is applicable in case of a beyond the design basis events.

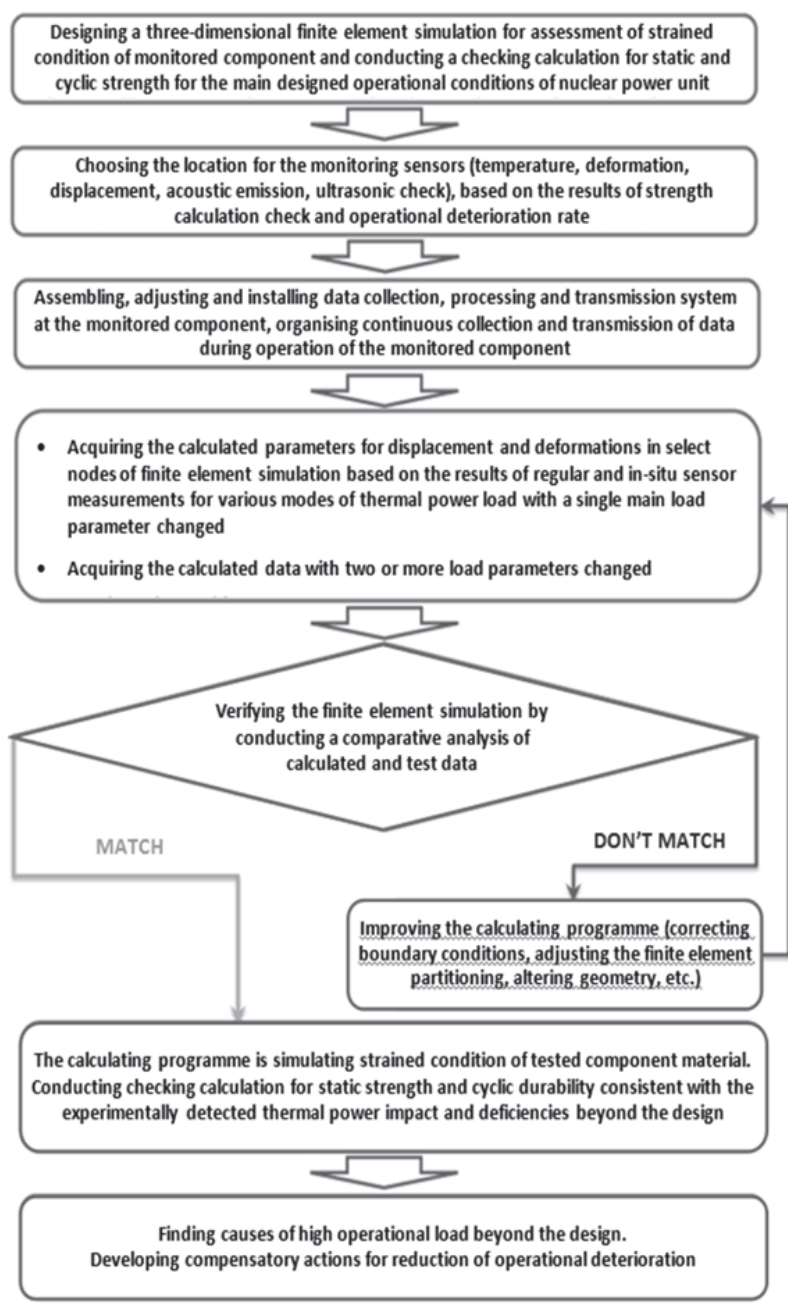

Fig. 4. Algorithm for the multi-parameter monitoring of condition and calculation analysis of operational deterioration rate.

The knowledge on operation of monitored equipment in various operation modes and corresponding analysis of the deflected mode and deficiency allow for the development of effective compensatory actions, aimed at 
reduction of main damaging factors' impact and improving durability of the monitored object. Moreover, it serves as the basis of control system data support for operation of both nuclear power units and automated repair systems. The use of set automated software and hardware complex in 2011 allowed to extend the service life of Novovoronezh Nuclear Power Plant Unit 5 on 30 years, as well as to assist the second service life extension of Unit 4 of the same power plant, which it currently undergoes.

\section{References}

1. V. Povarov, M. Bakirov, A. Danilov, Automatic system of multi-parameter monitoring of nuclear power installations status (Voronezh, Scientific book, 2017)

2. M. Bakirov, V. Povarov, A. Gromov, V. Levchuk, Izvestiya Wysshikh Uchebnykh Zawedeniy, Yadernaya Energetika, 3, 15-24 (2014)

3. M. Bakirov, V. Povarov, D. Nikolaev, A. Gromov, V. Levchuk, S. Gorokhov, Izvestiya Wysshikh Uchebnykh Zawedeniy, Yadernaya Energetika, 4, 5-10 (2014)

4. V. Povarov, M. Bakirov, A. Danilov, Voronezh technical university reporter, 6, 58-63 (2017)

5. A. Danilov, V. Golovnev, Digital control system

(Voronezh, VGLTA, 2007)

6. A. Danilov, Microprocessor elements and devices of local automatic (Voronezh, VGLTA, 2005)

7. A. Danilov, Technical means of automation (Voronezh, VGLTA, 2007)

8. A. Danilov, A. Pileich, Software distributed computing heterogeneous dynamic system parameters in real time (Voronezh, VGLTA, 2015)

9. V. Povarov, Nuclear Energy and Technology, 1, 6873 (2015)

10. M. Bakirov, V. Povarov, A. Gromov, V. Levchuk, Nuclear Energy and Technology, 1 (1), 32-36 (2015)

11. V. Povarov, M. Bakirov, Atomic Energy, 3, 153-162 (2016) 